\title{
Study of Plasma Shaping Effects on ITG Instability Using Global Gyrokinetic Code GKNET with Analytical Magnetic Equilibrium*)
}

\author{
Kenji IMADERA, Jhih-Yi LIN, Daichi NAKAJIMA and Yasuaki KISHIMOTO \\ Department of Fundamental Energy Science, Graduate School of Energy Science, \\ Kyoto University, Gokasho, Uji, Kyoto 611-0011, Japan
}

(Received 12 February 2020 / Accepted 13 October 2020)

\begin{abstract}
We extended the global gyrokinetic code GKNET (GyroKinetic Numerical Experiment of Tokamak) to noncircular shaped plasmas with analytical magnetic equilibria, which satisfy the Grad-Shafranov equation up to the second order with respect to aspect ratio. The extended version allows us to set the equilibria with non-unity elongation and non-zero triangularity, where the finite Shafranov shift is consistently determined. The allocated mesh follows the magnetic field line with periodic boundary conditions along the poloidal and toroidal directions so that the calculation cost for solving the gyrokinetic quasi-neutrality condition can be reduced by utilizing 1D FFT and MPI_ALLtoALL transpose technique. Based on the developed code, we studied the effect of elongation and triangularity on linear Ion Temperature Gradient (ITG) instability with adiabatic electrons in a non-circular shaped Tokamak by paying attention to the symmetry breaking of mode structure due to global profile effects. It is found that elongation reduces ITG instability owing to the effective reduction of flux-surface averaged ion temperature gradient and increases the asymmetry characterized by the Bloch angle $\theta_{b}$. On the other hand, when elongation is approximately unity, triangularity weakly affects the growth rate, while negative triangularity stabilizes ITG modes and increases the asymmetry in a large elongation regime.
\end{abstract}

(C) 2020 The Japan Society of Plasma Science and Nuclear Fusion Research

Keywords: global gyrokinetic simulation, elongation, negative triangularity, symmetry breaking, Bloch angle

DOI: $10.1585 /$ pfr. 15.1403086

\section{Introduction}

The control of plasma shaping is important to achieve high-performance plasmas, which sensitively influences on MHD instability. Some Tokamak experiments have shown that an increase in elongation and triangularity leads to the higher $\beta$ stability limit [1] with keeping higher confinement [2], indicating that plasma shaping affects microscale instability and associated turbulent transport [3-5] coupled with coherent macro-scale flows, such as zonal flow and Geodesic Acoustic Mode (GAM) [6].

By means of the rectangular $(R, Z, \zeta)$ version of our global gyrokinetic code GKNET [7,8], which uses a new real space field solver based on the diagonalization of zonal flow equation, we investigated plasma shaping effects on the collisionless damping of GAM. We found that not only elongation but also triangularity is effective in increasing the damping rate of GAM regardless of the sign, either plus or minus [9]. In this case, we employed the Miller equilibrium [10]. This equilibrium is a local parameterized model on a flux surface, which can be applied to local gyrokinetic analyses [3-5]. On the other hand, it does not globally satisfy the Grad-Shafranov equation so that elon-

author's e-mail: imadera@energy.kyoto-u.ac.jp

*) This article is based on the invited talk at the 36th JSPF Annual Meeting (2019, Kasugai). gation is assumed to be approximately unity, triangularity and Shafranov shift are small, which are independent from safety factor profile in our previous study [9]. This assumption limits the parameter regime for studying global plasma shaping effects.

From the numerical point of view, the rectangular version uses the fixed boundary condition in the poloidal plane and the allocated mesh points do not follow the magnetic flux surface. It usually increases the calculation cost for solving the gyrokinetic quasi-neutrality condition.

To resolve this problem, in this study, we introduced analytical magnetic equilibria that satisfy the GradShafranov equation up to the second order with respect to aspect ratio. Based on this version of GKNET, we studied the effect of elongation and triangularity on ITG instability with adiabatic electrons.

\section{Analytical Magnetic Equilibrium}

We employ the magnetic equilibrium with a noncircular plasma shape with non-unity elongation, non-zero triangularity and Shafranov shift [11], which is obtained by solving the Grad-Shafranov equation in the limit of small aspect ratio, i.e., $\varepsilon \equiv r / R_{0} \rightarrow 0$, by taking into account for plasma shape and extrapolating the equilibrium to $r=a_{0}$. 
Here, $r$ is the radial coordinate, $a_{0}$ and $R_{0}$ are the minor and major radii, respectively. The solution of the GradShafranov equation is then given by

$$
\begin{aligned}
R= & R_{0}+r \cos \theta-\Delta(r)+S_{2}(r) \cos \theta \\
& +S_{3}(r) \cos 2 \theta-S_{3}\left(a_{0}\right), \\
Z= & r \sin \theta-S_{2}(r) \sin \theta-S_{3}(r) \sin 2 \theta,
\end{aligned}
$$

where $\Delta(r)$ is the Shafranov shift, $S_{2}(r)$ and $S_{3}(r)$ are the shaping functions related to the elongation $\kappa$ and triangularity $\delta$, which are given by

$$
\begin{aligned}
\Delta(r)= & \int_{0}^{r} \Delta^{\prime}(\bar{r}) d \bar{r}, \\
\Delta^{\prime}(r)= & \frac{a_{0}^{2} q(r)^{2}}{2 q_{b} R_{0} r}\left[\frac{a_{0}^{2}}{q_{b} r^{2}} \log \left(\frac{q(r)}{q_{a}}\right)-\frac{1}{q(r)}\right] \\
& -\frac{R_{0} q(r)^{2}}{r^{3}} \int_{0}^{r} \bar{r}^{2} \beta^{\prime}(\bar{r}) d \bar{r}, \\
S_{2}(r)= & \frac{1-\kappa}{(1+\kappa)\left(3 q_{a}+q_{b}\right)}\left(\frac{q_{b}}{a_{0}^{2}} r^{3}+3 q_{a} r\right), \\
S_{3}(r)= & \frac{\delta}{4 a_{0}\left(2 q_{a}+q_{b}\right)}\left(\frac{q_{b}}{a_{0}^{2}} r^{4}+2 q_{a} r^{2}\right) .
\end{aligned}
$$

Here, we set a quadratic safety factor profile given by $q(r)=q_{a}+q_{b}\left(r / a_{0}\right)^{2}$. The contravariant components and covariant bases of magnetic field are given by

$$
\begin{aligned}
& B^{r}=0, B^{\theta}=\frac{\psi^{\prime}(r)}{\sqrt{g}}, B^{\zeta}=\frac{F(r)}{R^{2}}, \\
& b_{r}=\frac{g_{r \theta} \psi^{\prime}(r)}{\sqrt{g} B}, b_{\theta}=\frac{g_{\theta \theta} \psi^{\prime}(r)}{\sqrt{g} B}, b_{\zeta}=\frac{F(r)}{B},
\end{aligned}
$$

where

$$
\begin{aligned}
& \sqrt{g}=R\left(\frac{\partial R}{\partial r} \frac{\partial Z}{\partial \theta}-\frac{\partial R}{\partial \theta} \frac{\partial Z}{\partial r}\right), \\
& g_{\theta \theta}=\left(\frac{\partial R}{\partial \theta}\right)^{2}+\left(\frac{\partial Z}{\partial \theta}\right)^{2}, \\
& g_{r \theta}=\frac{\partial R}{\partial r} \frac{\partial R}{\partial \theta}+\frac{\partial Z}{\partial r} \frac{\partial Z}{\partial \theta}, \\
& B=\sqrt{g_{\theta \theta}\left(\frac{\psi^{\prime}(r)}{\sqrt{g}}\right)^{2}+\left(\frac{F(r)}{R}\right)^{2}}, \\
& F(r)=R_{0} B_{0}\left[1-\frac{r^{2}}{2 q(r)^{2} R_{0}^{2}}\left\{1+\frac{q(r)}{q_{a}}\right\}\right. \\
& \psi^{\prime}(r)=\frac{r F(r)}{q(r) R_{0}} .
\end{aligned}
$$

Here, $\beta(r)$ is the ion $\beta$-value given by $p(r) /\left[B_{0}^{2} / 2 \mu_{0}\right]\left(B_{0}\right.$ is the magnitude of magnetic field on the magnetic axis), which is assumed to be small because we consider the electrostatic limit in this study. Important input parameters in Eqs. (7) and (8) are the elongation $\kappa$, triangularity $\delta$, and the coefficients of safety factor profile, i.e., $q_{a}$ and $q_{b}$, while

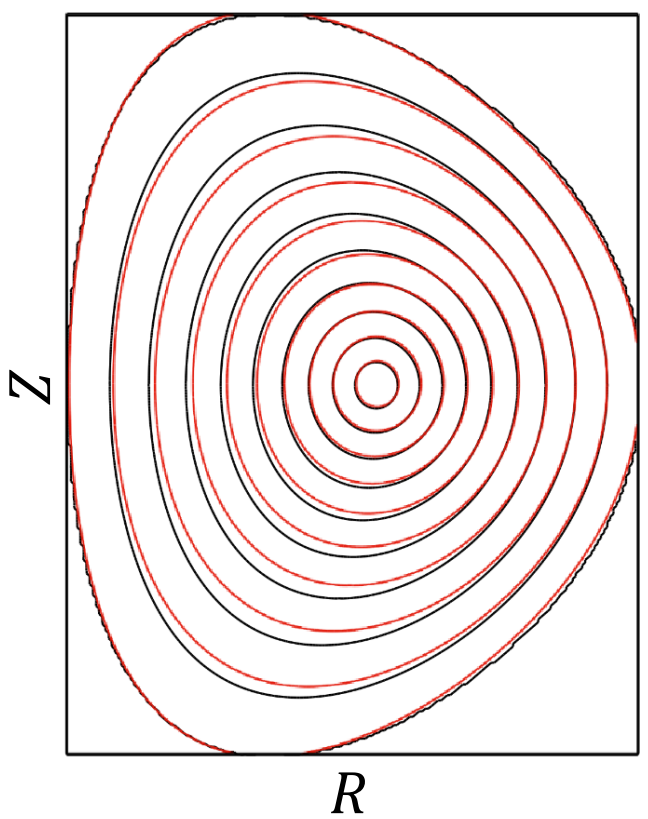

Fig. 1 Poloidal cross section of the magnetic surfaces in the case with $\beta\left(r=a_{0}\right)=0.01, \kappa=1.5$, and $\delta=0.3$. Red line shows an analytical solution given by Eqs. (1) - (14), and black line shows a numerical solution calculated by TASK/EQ.

the Shafranov shift is consistently given by the safety factor and pressure profiles, $q(r)$ and $p(r)$. Note that even in a concentric circular case with $\kappa=1$ and $\delta=0$, the magnetic flux is modified by the higher order correction terms in Eq. (13), which leads to the finite Shafranov shift. Figure 1 shows the typical shape of the magnetic surface in a poloidal cross section in the case with $a_{0} / R_{0}=0.36$, $\beta\left(r=a_{0}\right)=0.01, \kappa=1.5, \delta=0.3, q_{a}=0.85$, and $q_{b}=2.18$. Red line represents the analytical solution given by Eqs. (1) - (14), while black line represents that calculated numerically by TASK/EQ [12]. Since the analytical solution is obtained in the limit of $r / R_{0} \rightarrow 0$ and extrapolated to $r=a_{0}$, it is valid on both magnetic axis and last closed flux surface. On the other hand, the analytical solution has a small difference with numerical one in the other region. This originates from the higher order terms with respect to the aspect ratio in the Grad-Shafranov equation and the difference is found to be reduced by using a smaller aspect ratio.

\section{Gyrokinetic Equation System for Shaped Plasma}

Based on the model given by Eqs. (1) - (14), we newly derived the normalized gyrokinetic equation of motion as follows:

$$
\mathcal{J} \frac{d r}{d t}=v_{\|}\left(\frac{\partial b_{\zeta}}{\partial \theta}-\frac{\partial b_{\theta}}{\partial \zeta}\right) \frac{\partial H}{\partial v_{\|}}+\left(\frac{\partial b_{\zeta}}{\partial \theta}-\frac{\partial b_{\theta}}{\partial \zeta}\right) H
$$




$$
\begin{aligned}
& -\left(\frac{\partial H b_{\zeta}}{\partial \theta}-\frac{\partial H b_{\theta}}{\partial \zeta}\right) \\
\mathcal{J} \frac{d \theta}{d t}= & \sqrt{g} B^{\theta} \frac{\partial H}{\partial v_{\|}}+v_{\|}\left(\frac{\partial b_{r}}{\partial \zeta}-\frac{\partial b_{\zeta}}{\partial r}\right) \frac{\partial H}{\partial v_{\|}} \\
& +\left(\frac{\partial b_{r}}{\partial \zeta}-\frac{\partial b_{\zeta}}{\partial r}\right) H-\left(\frac{\partial H b_{r}}{\partial \zeta}-\frac{\partial H b_{\zeta}}{\partial r}\right) \\
\mathcal{J} \frac{d \zeta}{d t}= & \sqrt{g} B^{\zeta} \frac{\partial H}{\partial v_{\|}}+v_{\|}\left(\frac{\partial b_{\theta}}{\partial r}-\frac{\partial b_{r}}{\partial \theta}\right) \frac{\partial H}{\partial v_{\|}} \\
& +\left(\frac{\partial b_{\theta}}{\partial r}-\frac{\partial b_{r}}{\partial \theta}\right) H-\left(\frac{\partial H b_{\theta}}{\partial r}-\frac{\partial H b_{r}}{\partial \theta}\right) \\
\mathcal{J} \frac{d v_{\|}}{d t}= & -\sqrt{g}\left(B^{r} \frac{\partial H}{\partial r}+B^{\theta} \frac{\partial H}{\partial \theta}+B^{\zeta} \frac{\partial H}{\partial \zeta}\right) \\
& -v_{\|}\left[\frac{\partial}{\partial r}\left\{\left(\frac{\partial b_{\zeta}}{\partial \theta}-\frac{\partial b_{\theta}}{\partial \zeta}\right) H\right\}\right. \\
& +\frac{\partial}{\partial \theta}\left\{\left(\frac{\partial b_{r}}{\partial \zeta}-\frac{\partial b_{\zeta}}{\partial r}\right) H\right\} \\
& \left.+\frac{\partial}{\partial \zeta}\left\{\left(\frac{\partial b_{\theta}}{\partial r}-\frac{\partial b_{r}}{\partial \theta}\right) H\right\}\right]
\end{aligned}
$$

where $v_{\|}$is the parallel velocity along the magnetic field line, $\mu$ is the magnetic moment, $H=0.5 v_{\|}^{2}+\mu B+\langle\phi\rangle_{\alpha}$ is the gyrokinetic Hamiltonian $\left(\langle\phi\rangle_{\alpha}\right.$ is the gyro-averaged electrostatic potential), and $\mathcal{J} \equiv \sqrt{g} B_{\|}^{*}$ is the phase space Ja$\operatorname{cobian}\left(B_{\|}^{*}=B+v_{\|}\left[-\left(b_{\theta} / \sqrt{g}_{\theta \theta} R\right) \partial_{r} b_{\zeta}+\left(b_{\zeta} / r R\right) \partial_{r} b_{\theta}\right]\right.$ is the modified magnetic field). Here, all physical quantities are normalized as $r / \rho_{t 0 i} \rightarrow r, v_{t 0 i} t / \rho_{t 0 i} \rightarrow t, v_{\|} / v_{t 0 i} \rightarrow \bar{v}_{\|}$, $H / T_{i 0} \rightarrow H$ and $\boldsymbol{B} / B_{0} \rightarrow \overline{\boldsymbol{B}}\left(\rho_{t 0 i}\right.$ and $v_{t 0 i}$ are the ion gyro radius and ion thermal velocity at $r=0.5 a_{0}$, respectively). Note that the phase space volume conservation;

$$
\begin{aligned}
& \frac{\partial}{\partial r}\left(\mathcal{J} \frac{d r}{d t}\right)+\frac{\partial}{\partial \theta}\left(\mathcal{J} \frac{d \theta}{d t}\right)+\frac{\partial}{\partial \zeta}\left(\mathcal{J} \frac{d \zeta}{d t}\right)+\frac{\partial}{\partial v_{\|}}\left(\mathcal{J} \frac{d v_{\|}}{d t}\right) \\
& \quad=0
\end{aligned}
$$

is rigorously satisfied because Eqs. (15) - (18) are derived analytically from the gyrokinetic Hamiltonian incorporated with the condition $\nabla \cdot \boldsymbol{B}=0$ in the $(r, \theta, \zeta)$ coordinate system we defined in Sec. 2. This property is important for keeping numerical accuracy and stability in full- $f$ gyrokinetic simulations.

Based on Eqs. (1) - (18), we developed the new version of GKNET which is available for simulating non-circular shaped plasmas. The governing equation system consists of the gyrokinetic Vlasov equation for the full- $f$ ion distribution function and gyrokinetic quasi-neutrality condition with adiabatic electron in the $\boldsymbol{R}=(r, \theta, \zeta)$ coordinate system as

$$
\begin{aligned}
& \frac{\partial}{\partial t}(\mathcal{J} f)+\mathcal{J} \frac{d \mathbf{R}}{d t} \cdot \frac{\partial f}{\partial \mathbf{R}}+\mathcal{J} \frac{d v_{\|}}{d t} \frac{\partial f}{\partial v_{\|}}=0, \\
& -\nabla_{\perp} \cdot n(r) \nabla_{\perp} \phi+\frac{n(r)}{T_{e}(r)}\left[\phi-\langle\phi\rangle_{f}\right] \\
& =\iint\left\langle\delta f_{i}\right\rangle_{\alpha} \mathcal{J} d v_{\|} d \mu,
\end{aligned}
$$

where $f\left(\boldsymbol{R}, v_{\|}, \mu\right)$ is the gyro-center total ion distribution function, $n(r)$ and $T_{e}(r)$ are the flux-surface averaged den- sity and electron temperature, respectively. These quantities are normalized as $f v_{t i}^{3} / n_{0} \rightarrow f, n / n_{0} \rightarrow n, T_{e} / T_{i 0} \rightarrow$ $T_{e}\left(n_{0}\right.$ and $T_{i 0}$ are density and ion temperature at $r=0.5 a_{0}$, respectively). $\left\langle\delta f_{i}\right\rangle_{\alpha}$ is the gyro averaged perturbed distribution function and $\langle\phi\rangle_{f}$ is the flux-surface averaged electrostatic potential, which is obtained by taking the phase space Jacobian into account for the averaging [13]. Recently so called hybrid kinetic electron model has been introduced to GKNET, while we use the adiabatic electron model in this study as a first step to study magnetic shaping effects.

In this version of GKNET, we use 3D MPI decomposition for the $(r, \theta, \mu)$ domain. The spatial derivatives in Eq. (20) are discretized by using the fourth-order Morinishi scheme $[14,15]$ and the time integration is performed using the fourth-order explicit Runge-Kutta method. The magnetic field $\boldsymbol{B}$ is calculated from the vector potential $\boldsymbol{A}$ by using a fourth order finite difference method to numerically satisfy Eq. (19). Equation (21) is 1D Fouriertransformed along the $\zeta$ direction and then 1D Fouriertransformed along the $\theta$ direction after MPI_ALLtoALL transpose between the $\theta$ and $\zeta$ directions. Then by using MPI_ALLtoALL transpose between the $r$ and $\theta$ directions again, we can solve Eq. (19) in the $\left(r, k_{\theta}, k_{\zeta}\right)$ space, which has a tri-diagonal matrix form by applying the fourthorder finite difference method to the $r$ direction. Note that the matrix is not decomposed along the $r$ direction so that LU decomposition can be directly applied without any MPI communication. To take gyro-averaging for $\left\langle\delta f_{i}\right\rangle_{\alpha}$ and.$\langle\phi\rangle_{\alpha}$, we make the local Hermite interpolation on the poloidal plane to calculate the electrostatic potential on gyro ring and then take 20 sampling points average in real space without using the Bessel function in the $k$ space.

\section{Effect of Elongation and Triangu- larity on Linear ITG Instability}

By means of the linearized version of GKNET, we study the effect of elongation and triangularity on linear ITG instability. Here, we choose simulation parameters as $a_{0} / R_{0}=0.36, a_{0} / \rho_{t 0 i}=100, R_{0} / L_{n}=2.22$, $R_{0} / L_{T_{i}}=R_{0} / L_{T_{e}}=6.92$ at $r=0.5 a_{0}$. The safety factor profile is given by $q_{a}=0.85, q_{b}=2.18$. In this study, the ion $\beta$-value is assumed to be sufficiently small $\left(\beta\left(r=a_{0}\right)=0.001\right)$. We use the elongation and triangularity, which is linearly sheared in the $r$ direction as $\kappa=1+\left(\kappa_{0}-1\right) r / a_{0}$ and $\delta=\delta_{0} r / a_{0}$, respectively. Figure 2 shows the poloidal cross section of magnetic surfaces for $\kappa_{0}=1$ (gray) and $\kappa_{0}=1.5$ (red). It is observed that the plasma shape shrinks in the $R$ direction and stretches in the $Z$ direction by increasing $\kappa_{0}$. Here, to study plasma shaping effects on ITG mode, when we change the plasma shape, we also change the density and temperature profiles to fix their scale length at the half minor radius at the low field side given by $R_{0} / L_{n}\left(r=r_{r e f} \equiv R\left(r=0.5 a_{0}, \theta=0\right)-R_{0}\right)$ and $R_{0} / L_{T}\left(r=r_{r e f}\right)$, which is the main driving force of 
ITG mode. Figure 3 shows the typical 3D eigenfunction of the toroidal ITG mode with $n=15$ in the non-circular tokamak configuration with $\kappa_{0}=1.5$ and $\delta_{0}=0.3$. It is observed that the toroidal ITG mode twisted along the

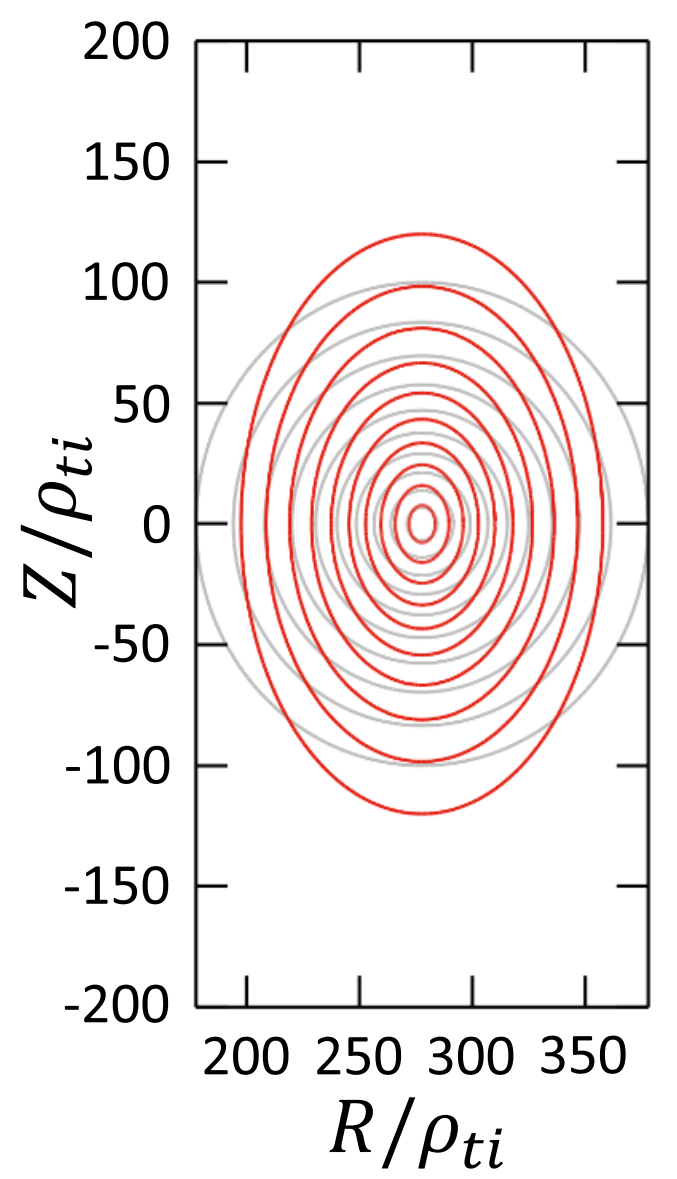

Fig. 2 Poloidal cross section of the magnetic surfaces in the cases with $\kappa_{0}=1$ (gray) and $\kappa_{0}=1.5$ (red). toroidal direction is excited around $r=r_{r e f}$ where the temperature gradient is the steepest and the corresponding poloidal mode number is found to be $m=21$, which satisfies the resonance condition, i.e., $q\left(r=r_{r e f}\right)=m / n=1.4$.

Figures 4 - 6 show the global eigenfunctions of the linear ITG mode with $n=15$ in the cases with three different triangularity, i.e., $\delta_{0}=0$ (Fig. 4 ), $\delta_{0}=0.3$ (Fig. 5), and $\delta_{0}=-0.3$ (Fig. 6). In each case, we set three different elongation: (a) $\kappa_{0}=1$, (b) $\kappa_{0}=1.2$ and (c) $\kappa_{0}=1.4$. It is observed that ballooning structures are located at the low field side in all cases, which results from the toroidal coupling of a series of eigenfunctions localized at each rational surface. In addition, it is observed that the structures exhibit an up-down asymmetry with respect to the mid-plane, which are tilted in the positive poloidal angle direction. The tilting angle is widely referred to as the Bloch angle $\theta_{b}$, which results from the first order profile effect with respect to the expansion ordering of ballooning representation in the toroidal system, i.e., $1 / n$, where $n$ is the toroidal mode number. In the ITG case with positive magnetic shear, since the radial shearing of diamagnetic drift frequency (i.e., $\partial \omega_{d} / \partial r$ ) is positive, the angle becomes a positive value according to the non-local ballooning theory [16]. In the case of $\left(\kappa_{0}, \delta_{0}\right)=(1,0)$ shown by Fig. 4 (a), the ITG mode is excited with the ballooning angle $\theta_{b}=0.49$, where the mode width of the ITG mode along the $r$ direction is the biggest. With an increase in elongation, the eigenfunction appears to be more stretched in the $Z$ direction and shrunken in the $R$ direction, as shown in Figs. 4 (b) and (c). Note that the ballooning angle is observed to increase to $\theta_{b}=0.68$ for $\kappa_{0}=1.2$ (Fig. 4 (b)) and $\theta_{b}=0.87$ for $\kappa_{0}=1.4$ (Fig. $4(\mathrm{c})$ ). This observation indicates that larger elongation provides stronger symmetry breaking of the mode structure owing to plasma shaping effects, which leads to larger intrinsic rotation [17].

Triangularity is also found to change the balloon-

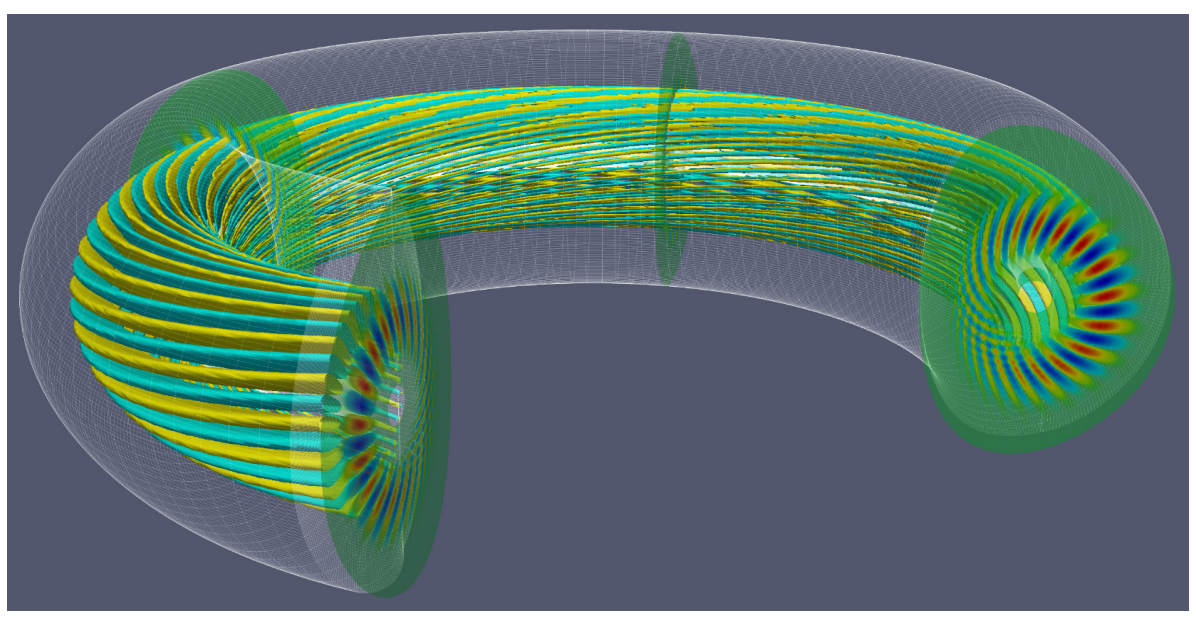

Fig. 3 Typical 3D eigenfunction of the toroidal ITG mode with $n=15$ in the non-circular tokamak configuration with $\beta\left(r=a_{0}\right)=0.01$, $\kappa_{0}=1.5$, and $\delta_{0}=0.3$. 

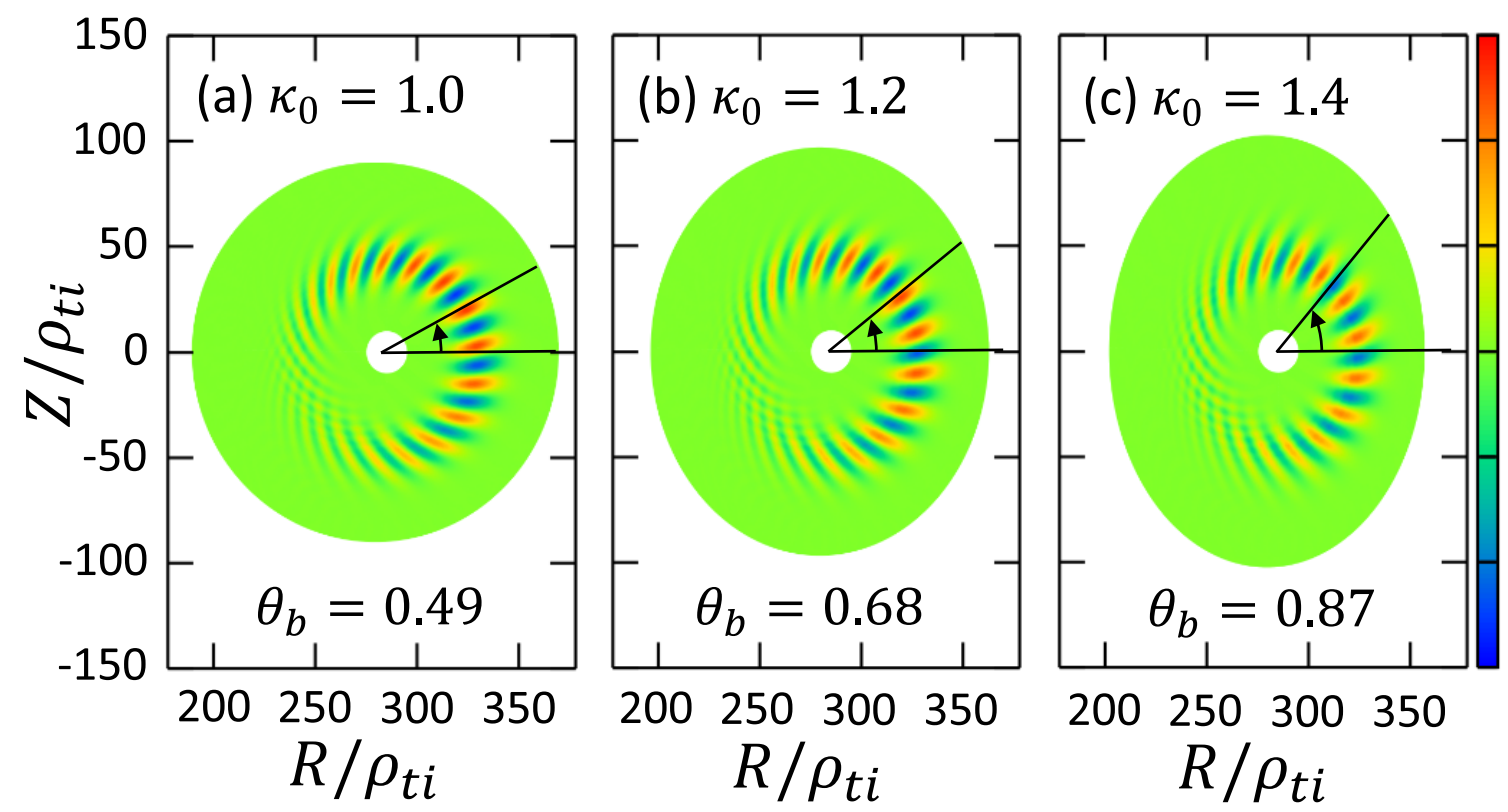

Fig. 4 Global eigenfunctions of the linear ITG mode in the cases with $\delta_{0}=0$. (a) $\kappa_{0}=1$, (b) $\kappa_{0}=1.2$, and (c) $\kappa_{0}=1.4$ are used as elongation.

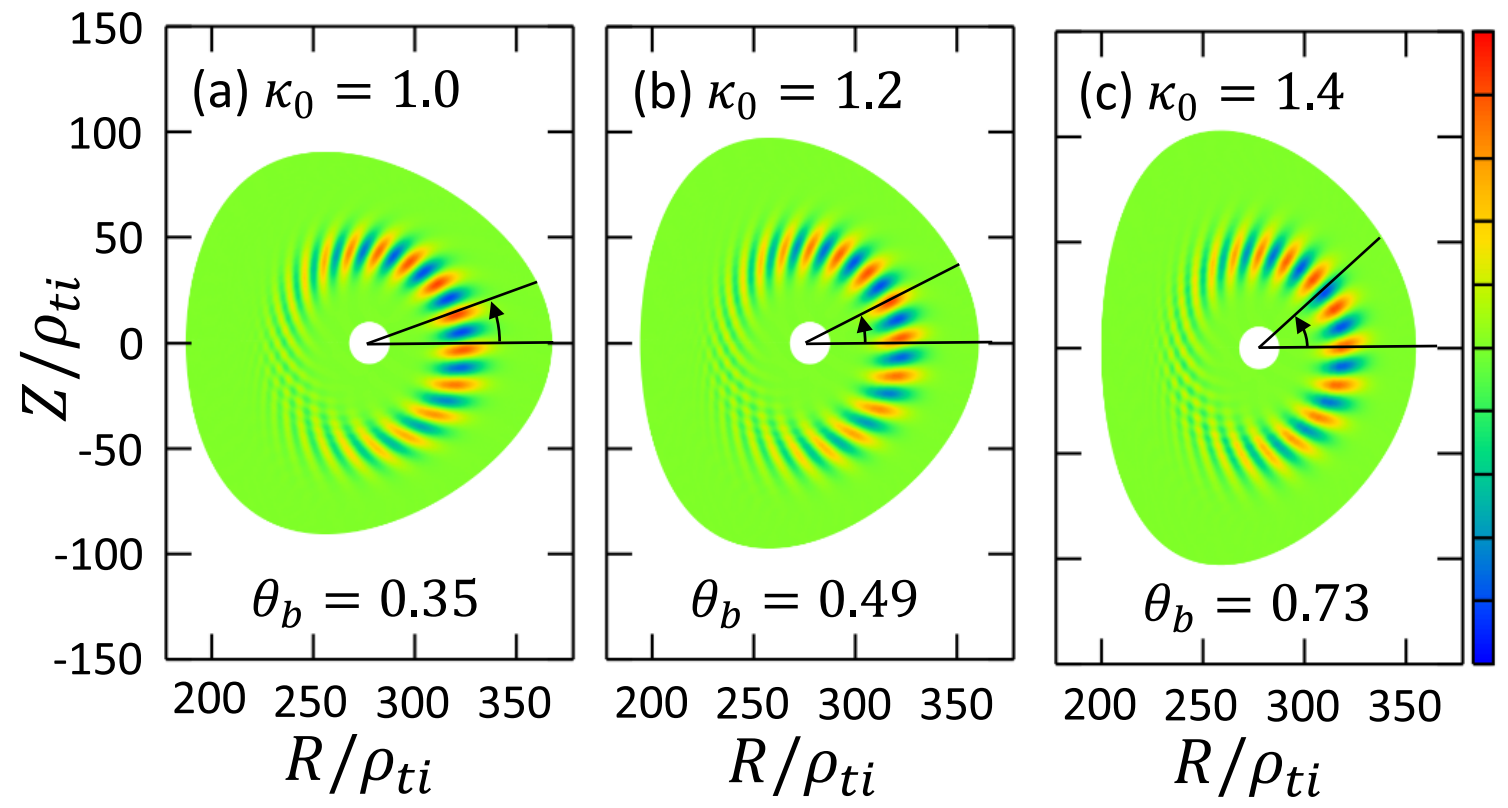

Fig. 5 Global eigenfunctions of the linear ITG mode in the cases with $\delta_{0}=0.3$. (a) $\kappa_{0}=1$, (b) $\kappa_{0}=1.2$, and (c) $\kappa_{0}=1.4$ are used as elongation.

ing structure and the corresponding ballooning angle, as shown in Figs. 5 - 6. Especially in the $\kappa_{0}=1.4$ case, the structure becomes clearly tilted with $\theta_{b}=1.04$ in the negative triangularity case (Fig. 6 (c)) than that with $\theta_{b}=0.73$ in the positive case (Fig. 5 (c)). This is related to the fact that while the positive triangularity stretches the temperature profile in the high field side (see Fig. 5), the negative one stretches the profile in the low field side (see Fig. 6), which provides bigger symmetry breaking of ITG modes.
Figure 7 shows the relationship between the growth rate of linear ITG mode and $\delta_{0}$ in the cases with $\kappa_{0}=1$ (black), $\kappa_{0}=1.2$ (red), and $\kappa_{0}=1.4$ (green). It is found that the ITG mode is stabilized by elongation, which rates are $\gamma\left(\kappa_{0}=1.4, \delta_{0}=0\right) / \gamma\left(\kappa_{0}=1, \delta_{0}=0 \sim 0.61\right.$, $\gamma\left(\kappa_{0}=1.4, \delta_{0}=0.3\right) / \gamma\left(\kappa_{0}=1, \delta_{0}=0.3\right) \sim 0.70$, and $\gamma\left(\kappa_{0}=1.4, \delta_{0}=-0.3\right) / \gamma\left(\kappa_{0}=1, \delta_{0}=-0.3\right) \sim 0.47$, respectively. Since $R_{0} / L_{T}\left(r=r_{r e f}, \theta=0\right)$ at the midplane is fixed in this study, the elongated shape effec- 


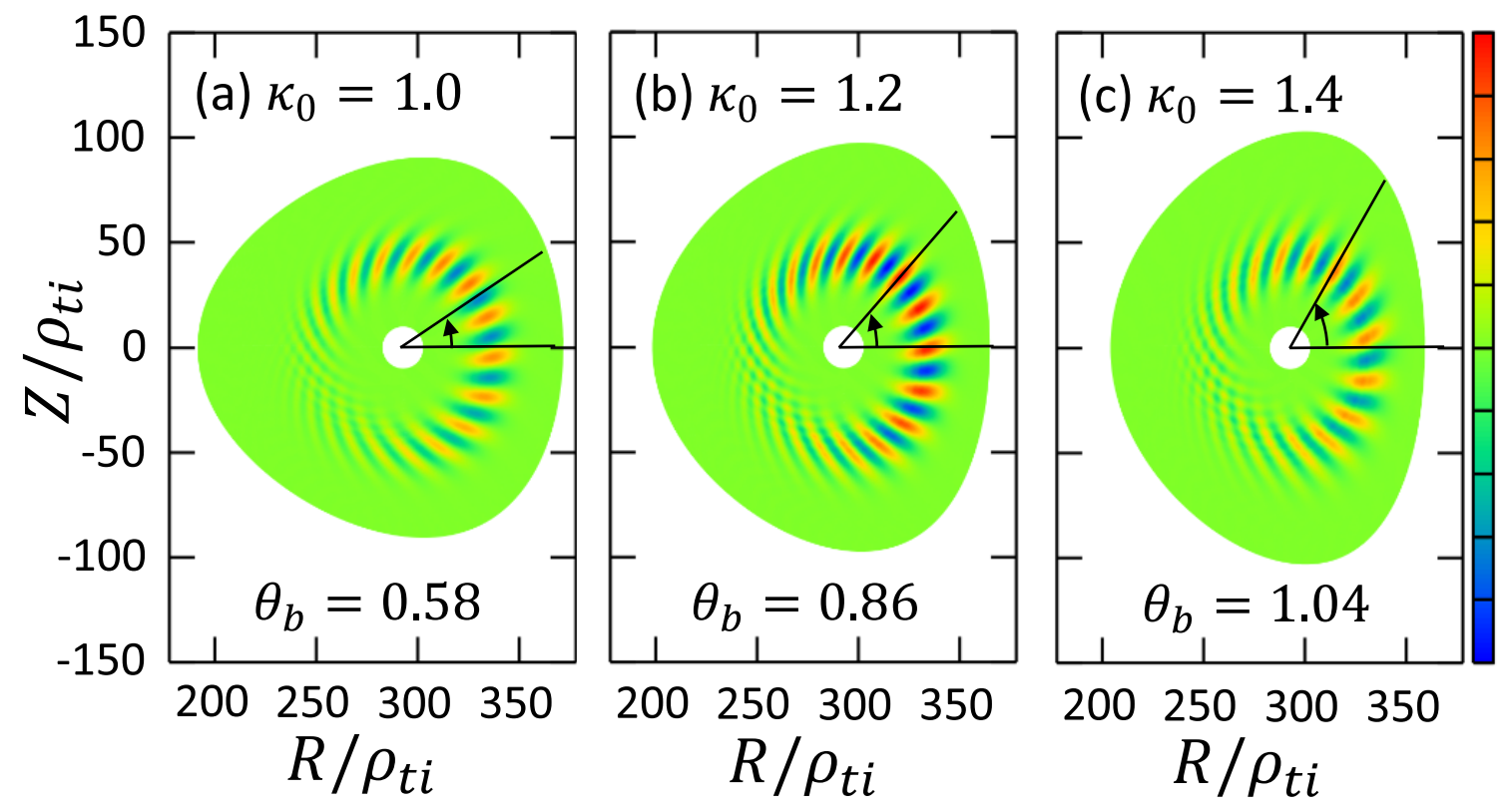

Fig. 6 Global eigenfunctions of the linear ITG mode in the cases with $\delta_{0}=-0.3$. (a) $\kappa_{0}=1$, (b) $\kappa_{0}=1.2$, and (c) $\kappa_{0}=1.4$ are used as elongation.

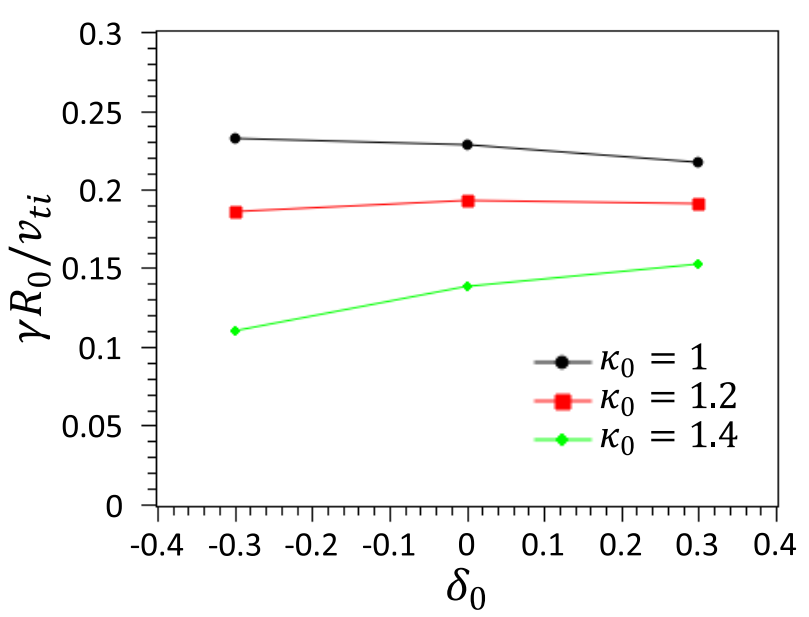

Fig. 7 Relationship between the normalized growth rate of the linear ITG mode and $\delta_{0}$ in the cases with $\kappa_{0}=1$ (black), $\kappa_{0}=1.2(\mathrm{red})$, and $\kappa_{0}=1.4$ (green).

tively stretches the temperature profile because the interval of magnetic flux surface becomes sparse than that of magnetic flux at the mid-plane. This means that the fluxsurface averaged temperature gradient, which is the effective driving force of ITG modes, is also reduced. This is the main reason why the elongation shows the stabilizing effect. The same idea can be applied to the effect of triangularity. The flux-surface averaged plasma width at the low field side $(-\pi / 2<\theta<\pi / 2)$, namely $\bar{w}=1 / \pi \int_{-\pi / 2}^{\pi / 2} w(\theta) d \theta$ is $\bar{w}(\kappa=1.4, \delta=0.3)=94.9 \rho_{t 0 i}$ and $\bar{w}(\kappa=1.4, \delta=-0.3)=98.8 \rho_{t 0 i}$, respectively $(w(\theta)$ is the plasma width at each poloidal angle). The gap of $\bar{w}$ between positive and negative triangularity cases becomes smaller when elongation reaches unity. This means that the negative triangularity in a large elongation regime can stretch the temperature profile in the low field side where the ITG mode is excited, which leads to the bigger stabilization effect. The global profile shearing, which originates from the inhomogeneity of background profiles, also stabilize the ITG instability but as a next order [16] and the effect of Shafranov shift on linear ITG instability in the electrostatic limit is considered to be small [18] so that it is natural to conclude that the effective reduction of temperature gradient stabilizes ITG modes.

\section{Summary}

We extended the GKNET code to non-circular shaped plasmas with analytical magnetic equilibria, which satisfy the Grad-Shafranov equation up to the second order with respect to aspect ratio and allows us to set the consistent elongation, triangularity, and Shafranov shift. In this version of GKNET, the phase space conservation of the gyrokinetic Vlasov equation is analytically and numerically fulfilled with smaller calculation cost for solving the gyrokinetic quasi-neutrality condition by utilizing 1D FFT and MPI_ALLtoALL transpose technique, so that it has a capability for long-time and stable simulation of noncircular shaped plasmas.

Based on this version, we studied the effect of elongation and triangularity on linear ITG instability with adiabatic electrons. It is found that elongation reduces ITG instability owing to the effective reduction of flux-surface averaged ion temperature gradient and increases the asymmetry characterized by the Bloch angle $\theta_{b}$. On the other hand, when elongation is approximately unity, triangular- 
ity weakly affects the growth rate, while negative triangularity stabilizes ITG modes and increases the asymmetry in a large elongation regime.

We have been also introducing a numerical magnetic equilibrium calculated by TASK/EQ, which allows a more rigorous treatment of magnetic equilibrium and comparison with experiments. These results will be reported in another manuscript in the near future.

Another future study is the flux-driven simulation of a non-circular shaped plasma using the full- $f$ version of GKNET. Since the temperature gradient at the mid-plane is fixed in this linear study, elongation and triangularity can change temperature gradient, which indirectly stabilizes/destabilizes ITG modes. To study their direct stabilization effect, flux-driven simulation is one candidate because we can judge their stabilization effect from the selfconsistently established temperature profile, which is insensitive to the initial one, under the power balance.

The other future work is to introduce the selfconsistent temporal evolution of magnetic equilibrium. In full-f simulation with kinetic or hybrid electrons, the bootstrap current is precisely taken into account, which can modify the safety factor profile and resultant magnetic equilibrium in time. The temporal evolutions of density/rotation/pressure profiles can also change the equilibrium. By reflecting such effects to the analytical magnetic equilibrium, we can do a quasi-electromagnetic full$f$ simulation, which can help us to understand the role of high bootstrap fraction on internal transport barrier formation [19].

\section{Acknowledgments}

Authors would like to thank Drs. Masatoshi Yagi,
Haruki Seto, and Wang Wei in QST and Dr. Kevin Obrejan in JAEA for their valuable comments. The simulations were performed on the JFRS-1 (IFERC-CSC) and Plasma Simulator (NIFS). This work was supported by JSPS KAKENHI Grant Number 16K17844 and the Joint Special Design Team Collaborative Research Program (31K031).

[1] J.G. Cordey et al., Nucl. Fusion 45, 1078 (2005).

[2] F. Troyon et al., Plasma Phys. Control. Fusion 26, 209 (1984).

[3] J.E. Kinsey et al., Phys. Plasmas 14, 102306 (2007).

[4] E.A. Belli et al., Phys. Plasmas 15, 092303 (2008).

[5] P. Angelino et al., Phys. Rev. Lett. 102, 195002 (2009).

[6] Y. Xiao and P.J. Catto, Phys. Plasmas 13, 082307 (2006).

[7] K. Imadera et al., Proc. of 26th IAEA Fusion Energy Conf., TH/P3-3 (2016).

[8] K. Obrejan et al., Plasma Fusion Res. 10, 3403042 (2015).

[9] K. Obrejan et al., Comput. Phys. Commum. 216, 8 (2017).

[10] R.L. Miller et al., Phys. Plasmas 5, 973 (1998).

[11] J.P. Graves, Plasma Phys. Control. Fusion 55, 074009 (2013).

[12] A. Fukuyama et al., Proc. of 20th IAEA Fusion Energy Conf., IAEA-CSP-25/TH/P2-3 (2004).

[13] V. Grandgirard et al., Comput. Phys. Commun. 207, 35 (2016).

[14] Y. Morinishi et al., J. Comput. Phys. 143, 90 (1998).

[15] Y. Idomura et al., J. Comput. Phys. 226, 224 (2007).

[16] Y. Kishimoto et al., Plasma Phys. Control. Fusion 41, A663 (1999).

[17] Y. Camenen et al., Nucl. Fusion 51, 073039 (2011).

[18] A. Ishizawa et al., Phys. Rev. Lett. 123, 025003 (2019).

[19] G.M. Staebler et al., Nucl. Fusion 58, 115001 (2018). 